\title{
A NOTE ON WALLMAN SPACES
}

\section{ALAN ZAME}

In [3] Orrin Frink introduced the notion of a Wallman compactification of a Tychonoff space. A. K. and E. F. Steiner [6] have recently shown that any compact metric space (or product of compact metric spaces) is a Wallman compactification of each of its dense subspaces. Their result is an immediate consequence of earlier work and a theorem they proved about the existence of a certain kind of base of closed sets in a compact metric space. The purpose of this note is to give a different proof of this latter result, obtaining as a consequence a slightly stronger version.

Definition. Let $X$ be a Tychonoff space. A family $\mathrm{Z}$ of closed subsets of $X$ is said to be a regular normal base for $X$ if $\mathrm{Z}$ is a base for the closed subsets of $X$, if each member of $Z$ is a regular set (the closure of its interior) and if

(i) $\mathrm{Z}$ is closed under finite unions and intersections; (i.e., $\mathrm{Z}$ is a ring)

(ii) if $x \in X, F$ a closed subset of $X, x \notin F$, then there exists $Z \in Z$ such that $x \in Z \subset X \backslash F$;

and

(iii) if $A, B \in \mathrm{Z}, A \cap B=\varnothing$ then there exists $C, D \in \mathcal{Z}$ such that

$$
A \subset X \backslash C, \quad B \subset X \backslash D \text { and }(X \backslash C) \cap(X \backslash D)=\varnothing .
$$

If $X$ is a compact metric space, then (ii) and (iii) will hold for $\mathbb{Z}$ if $Z$ is a base for closed sets and is a ring.

Our result is the following

TheOREM. Let $X$ be a compact metric space and let $R$ be any countable ring of regular closed subsets of $X$. Then there is a regular normal base $\mathbb{Z}$ for $X$ such that $\mathrm{Z} \supseteq \Omega$.

We notice that the result may fail if $R$ is not countable. For instance, let $X=[0,1], R=\{[0, \alpha]: 0<\alpha \leqq 1\}$. Then any closed set not containing 0 must intersect some member of $Q$ in a single point and $R$ can not be extended to a normal base for $X$.

Proof of the Theorem. From now on, let $X$ be a compact metric space. Let $I=[0,1]$ and let $P=\prod_{i=1}^{\infty} I$. We may assume that $X \subset P$. If $p \in P$ we will write $p=\left(p_{1}, p_{2}, \cdots\right)$.

Let $Z^{+}$denote the set of positive integers. Let $\mathcal{F}$ denote the set of all functions whose domains are subsets of $Z^{+}$and whose ranges are

Received by the editors July $19,1968$. 
contained in $I$. (For convenience, we will also include the "function" $\psi$ whose domain is empty.) If $\alpha$ is such a function then $|\alpha|$ will denote the domain of $\alpha$ and $\#|\alpha|$ the number of elements in $|\alpha|$ if it is finite. If $\alpha$ and $\beta$ are such that $|\alpha| \cap|\beta|=\varnothing$, then by $\alpha \bigvee \beta$ we will mean the function whose domain is $|\alpha| \cup|\beta|$ and which is defined in the obvious manner. We will also identify points in $P$ with functions in $\mathcal{F}$ whose domains are $Z^{+}$. If $\alpha \in \mathcal{F}$ and $\sharp|\alpha|=n$, we will write $|\alpha|$ $=\left\{\bar{\alpha}_{1}, \bar{\alpha}_{2}, \cdots, \bar{\alpha}_{n}\right\}$ where $\bar{\alpha}_{1}<\bar{\alpha}_{2}<\cdots<\bar{\alpha}_{n}$.

Let $S$ denote the set of all finite sequences of \pm 1 . If $s \in S$, then \#s will denote the length of $s$. Let $\alpha \in \mathcal{F}$ with $\#|\alpha|=n$ and $s \in \mathcal{S}$ with $\# s=n$. The quadrant $Q(\alpha, s)$ determined by $\alpha$ and $s$ is defined by (where $s=\left(s_{1}, \cdots, s_{n}\right)$ )

$$
Q(\alpha, s)=\left\{y \in P: s_{j} y_{\bar{\alpha}_{j}}>s_{j} \alpha\left(\bar{\alpha}_{j}\right) \text { for } 1 \leqq j \leqq n\right\} .
$$

If $\sigma$ is the sequence of length 0 , then by the above definition, or by convention, $Q(\psi, \sigma)=P$.

Let $\alpha \in \mathcal{F}$ with $|\alpha|$ finite. Then $\alpha$ is admissible for a closed subset $B$ of $P$ if for every $\beta \in \mathcal{F},|\alpha| \cap|\beta|=\varnothing,|\alpha| \cup|\beta|=Z^{+}$such that $\alpha \bigvee \beta \in B$ and for every neighborhood $N$ (relative to $P$ ) of $\alpha \bigvee \beta$ and every $s \in S$ with $\# s=\#|\alpha|$ we have $B \cap N \cap Q(\alpha, s) \neq \varnothing$. Notice that $\psi$ is admissible.

The basic result is the following:

Lemma. Let $\alpha \in \mathcal{F}$ be admissible for $B$. Let $k \notin|\alpha|$ be a fixed positive integer. Then the set of $\beta \in \mathcal{F}$ with $|\beta|=\{k\}$ such that $\alpha \bigvee \beta$ is not admissible for $B$ is countable. In particular, the set of $\alpha$ with $\#|\alpha|=1$ which are not admissible for $B$ is countable.

We will now show that the lemma implies our theorem. Let $R$ be a countable regular ring. By induction and the use of our lemma applied to $X$ and the members of $R$ we may prove the existence of countable sets $D_{1}, D_{2}, \cdots, D_{n}, \cdots$ such that

(i) for each $j, D_{j} \subset \mathcal{F}$ and if $\alpha \in D_{j}$ then $|\alpha|=\{j\}$;

(ii) $\left\{\alpha(j): \alpha \in D_{j}\right\}$ is dense in $I$ for each $j$;

(iii) if $\alpha \in D_{n_{1}} \vee \cdots \vee D_{n_{k}}\left(n_{i} \neq n_{j}\right.$ if $\left.i \neq j\right)$ then $\alpha$ is admissible for $X$ and for each $B \in R$.

For each $j$ we may write $D_{j}=D_{j}^{\prime} \cup D_{j}^{\prime \prime}$ where $D_{j}^{\prime} \cap D_{j}^{\prime \prime}=\varnothing$ and both $\left\{\alpha(j): \alpha \in D_{j}^{\prime}\right\}$ and $\left\{\beta(j): \beta \in D_{j^{\prime \prime}}\right\}$ are dense in $I$. Let $\mathcal{R}_{j}$ $=\left\{[\alpha(j), \beta(j)]: \alpha \in D_{j}^{\prime}, \beta \in D_{j}^{\prime \prime}\right\}$. Let

$$
\Omega_{1}=\left\{\prod_{j=1}^{\infty} E_{j}: E_{j}=(-\infty, \infty)\right.
$$

for all but finitely many $j$ and $E_{j}=\Re_{j}$ for the remaining $\left.j\right\}$. 
Let $\tilde{\mathfrak{Q}}_{2}$ consist of all finite unions of members of $\tilde{\mathscr{Q}}_{1}$ and let $\mathrm{Z}=$ the ring generated by $R \cup\left\{E \cap X: E \in \tilde{\mathbb{R}}_{22}\right\}$. That $\mathcal{Z}$ is a normal base for $X$ follows easily from the density of $\left\{\alpha(j): \alpha \in D_{j}^{\prime}\right\}$ and $\left\{\beta(j): \beta \in D_{j}^{\prime \prime}\right\}$ for all $j$. That the members of $\mathcal{Z}$ are regular follows from the admissibility of all $\alpha \in D_{n_{1}} \vee \cdots \vee D_{n_{k}}$ for $X$ and for all $B \in R$ and the fact that if $A$ and $B$ are regular and $A \cap B$ is regular in $B$ then $A \cap B$ is regular.

We now turn to the proof of the lemma. Let $\alpha$ be admissible and let $k \notin|\alpha|$ be a positive integer. Suppose $\#|\alpha|=n$. Let $e>0$, $s \in \mathcal{S}$ with $\# s=n+1$ and an integer $m \geqq \max \left(\bar{\alpha}_{n}, k\right)$ be fixed. For simplicity of notation, assume that the sequence $s$ is indexed with the elements of $|\alpha| \cup\{k\}$ in increasing order. Let $\tilde{s}$ be the sequence $s$ with the term $s_{k}$ (under this new labelling) deleted, and assume that $s_{k}=+1$. For $u \in P$ let

$$
C_{u}^{e}=\left\{y \in P:\left|u_{i}-y_{i}\right|<e, i=1,2, \cdots, m\right\} .
$$

Suppose there exists $\beta \in \mathcal{F}$ with $|\beta|=\{k\}$ such that

(i) there exists $\gamma \in \mathcal{F}$ with $|\gamma| \cap(|\alpha| \cup\{k\})=\varnothing$ and $|\alpha| \cup\{k\}$ $\cup|\gamma|=Z^{+}$, such that $p=\alpha \bigvee \beta \bigvee \gamma \in B$, and

(ii) $B \cap C_{p}^{e} \cap Q(\alpha \vee \beta, s)=\varnothing$.

We claim that the set of $\operatorname{such} \beta$ is finite. This will show that the number of $\beta$ such that $\alpha \bigvee \beta$ is not admissible is countable. Suppose $\beta^{\prime} \neq \beta$ is such that there exists $\gamma^{\prime}$ as in (i) such that $p^{\prime}=\alpha \bigvee \beta^{\prime} \bigvee \gamma^{\prime}$ satisfies (i) and (ii) above. We claim that $C_{p}^{e / 4} \cap C_{p^{\prime}}^{e / 4}=\varnothing$. This will clearly prove the result.

Suppose that $q \in C_{p}^{e / 4} \cap C_{p}^{e / 4}$. Assume that $\beta(k)>\beta^{\prime}(k)$. Then $\left|\beta(k)-q_{k}\right|<e / 4$ and $\left|\beta^{\prime}(k)-q_{k}\right|<e / 4$ so that $\left|\beta(k)-\beta^{\prime}(k)\right|<e / 2$. Let $\delta=\beta(k)-\beta^{\prime}(k)$. Then $0<\delta<e / 2$. Since $\alpha$ is admissible, there exists $r \in B \cap Q(\alpha, \tilde{s}) \cap C_{p}^{\delta}$. We claim that $r \in B \cap C_{p^{\prime}}^{e} \cap Q\left(\alpha \bigvee \beta^{\prime}, s\right)$, in contradiction of (ii) above, thus proving the result. Certainly $r \in B$. Furthermore, since $r \in Q(\alpha, \tilde{s})$ and $\left|r_{k}-\beta(k)\right|<\delta=\left|\beta(k)-\beta^{\prime}(k)\right|$ we certainly have that $r_{k}>\beta^{\prime}(k)$ so that $r \in Q\left(\alpha \bigvee \beta^{\prime}, s\right)$. Finally, to show that $r \in C_{p^{\prime}}^{e}$ we have to show that $\left|r_{j}-p_{j}^{\prime}\right|<e$ for $j=1, \cdots, m$. But $\left|r_{j}-p_{j}\right|<\delta$ and

$$
\left|p_{j}-p_{j}^{\prime}\right| \leqq\left|p_{j}-q_{j}+q_{j}-p_{j}^{\prime}\right|<e / 4+e / 4=e / 2,
$$

so the result follows by the triangle inequality.

\section{REFERENCES}

1. Richard A. Alo and H. L. Shapiro, $A$ note on compactifications and semi-normal spaces, Austral. J. Math. 8 (1968), 104-114. 
2. - Normal bases and compactifications, Math. Ann. 175 (1968), 337-340.

3. Orrin Frink, Compactifications and semi-normal spaces, Amer. J. Math. 86 (1964), 602-607.

4. E. F. Steiner, Wallman spaces and compactifications, Fund. Math. 61 (1968), 295-303.

5. A. K. and E. F. Steiner, Wallman and Z-compactifications, Duke Math. J. 35 (1968), 269-276.

6. - Products of compact metric spaces are regular Wallman, Indag. Math. (to appear).

UNIVERSITY OF MiAMI 